Nervenarzt 2020 • 91:936-945

https://doi.org/10.1007/s00115-020-00967-0

Online publiziert: 28 . Juli 2020

(c) Der/die Autor(en) 2020

\section{Hintergrund}

Demenzen sind für betroffene Patienten und ihre Angehörigen in der Regel mit erheblichen medizinischen, prognostischen und sozioökonomischen Folgen verbunden. Unsere Gesellschaft stellen sie vor große gesundheitsökonomische und gesellschaftspolitische Probleme [1]. Trotz der zunehmend gesünderen Lebensführung und der damit assoziierten Risikoreduktion, an einer Demenz zu erkranken, ist es aufgrund des demographischen Wandels in den vergangenen Jahrzehnten zu einem rasanten Anstieg der Anzahl an Patienten mit einer Demenz gekommen. Diese Entwicklung wird sich in den kommenden Jahrzehnten fortsetzen [1, 8]. Demenzen stellen typischerweise Erkrankungen des höheren Lebensalters dar; das Alter ist der bedeutendste Risikofaktor für die Entstehung einer Demenz. Die Alzheimer-Krankheit ist sowohl bei älteren als auch bei jüngeren Patienten die mit Abstand häufigste Ursache einer Demenz. Die jährliche Inzidenzrate einer Demenz steigt von etwa $0,5 \%$ im Alter von 65 bis 70 Jahre auf 6-8\% bei Menschen > 85 Jahre $[1,29]$. Dennoch ist es wichtig, Demenzen und neurodegenerative Erkrankungen auch bei jüngeren Patienten als Differenzialdiagnose bei alltagsrelevanten kognitiven Defiziten zu bedenken, da sich etwa $8 \%$ aller Demenzen vor dem 65. Lebensjahr manifestieren [10]. Diagnostik und Management von jüngeren Patienten mit demenziellen Syndromen stellen im klinischen Alltag große Herausforderungen dar, da deren Epidemiologie und klinische Präsentation in unterschiedlichen Altersgruppen erheblich variieren. Zudem ist das differenzialdiagnostische Spektrum bei Jüngeren deutlich breiter [10,31]. In diesem

\author{
Matthias Pawlowski · Andreas Johnen - Thomas Duning ${ }^{1}$ \\ ${ }^{1}$ Klinik für Neurologie mit Institut für Translationale Neurologie, Universitätsklinikum Münster, Münster, \\ Deutschland
}

\title{
Früh beginnende Demenzen
}

Artikel geben wir einen Überblick über die häufigsten Ursachen von Demenzen bei Patienten <65 Jahre und vermitteln ein alltagspraktisches und standardisiertes diagnostisches Vorgehen.

\section{Definitionen und Epidemiologie}

In der aktuellen S3-Leitlinie wird der Begriff Demenz - angelehnt an die ICD10Klassifikation - klinisch definiert, d.h. als eine progrediente kognitive Störung mit alltagsrelevanter Ausprägung. Nach dem Feststellen dieses Symptomkomplexes wird eine weiterführende Diagnostik empfohlen, um die Ätiologie der Symptome festzustellen. Nicht neurodegenerative Erkrankungen sollten als Ursache ausgeschlossen werden. In der Revision der Leitlinie aus dem Jahr 2016 wird jedoch schon auf die aktuellen Entwicklungen der internationalen Diagnosekriterien hingewiesen, insbesondere auf die Sensitivität spezifischer laborchemischer Biomarker, wie z. B. Amyloid- oder TauProtein-Werte. Zudem werden die Erkrankungen als fortschreitendes Kontinuum betrachtet, deren Pathophysiologie sich langsam-progredient und meist schon Jahre oder Dekaden vor Symptombeginn zeigt, d.h. eine Unterteilung in z. B. Alzheimer-Demenz oder klinische Vorstufen findet nicht mehr statt, sondern nur noch die Definition AlzheimerKrankheit.

Anhand des Manifestationsalters werden Demenzen recht willkürlich bzw. vorwiegend aus psychosozialen Gründen dichotomisiert in solche, die vor oder nach dem 65. Lebensjahr auftreten, wobei sich in der englischsprachigen Literatur hierfür die Begriffe „young onset dementia“bzw. „late onset dementia“ etabliert haben [19]. Die früher verwandten Begriffe „präsenil“ bzw. „senil“ sollten heute keine Anwendung mehr finden, da sie suggerieren, dass alltagsrelevante kognitive Störungen bei älteren Menschen eine normale Entwicklung sind. Früh beginnende Demenzen umfassen daher per definitionem alle Demenzen mit einem Erkrankungsalter zwischen dem 18. und 65. Lebensjahr.

Es existieren kaum breit angelegte, epidemiologische Studien zur Prävalenz und Inzidenz früh beginnender Demenzen. Die untersuchten Fallzahlen sind klein und die eingeschlossenen Altersgruppen, Populationen, Methoden und Diagnosekriterien in den jeweiligen Studien deutlich verschieden [21]. In den vier größten, bevölkerungsbezogenen Registerstudien betrug die Prävalenz bei den 45 - bis 64Jährigen 81-143/100.000 Einwohner [14, $16,20,35]$, wobei sich eine altersabhängige exponentielle Zunahme der Prävalenz zeigte (• Abb. 1a). Das durchschnittliche Manifestationsalter aller Demenzen, die sich vor dem 65 . Lebensjahr manifestieren, beträgt 58 Jahre [10, 31]. Obwohl Demenzen bei älteren Menschen deutlich häufiger sind, macht der Anteil jüngerer Patienten in Gedächtnisambulanzen und spezialisierten Kliniken bis zu einem Drittel der Fälle aus [4].

\section{Das ätiologische Spektrum früh beginnender Demenzen}

Die Ursachen der Demenzen können grob unterteilt werden in primär neurodegenerative Erkrankungen, vaskuläre Demenzen und das breite Spektrum der sekundären Demenzen. Die primär neurodegenerativen Erkrankungen sind nicht nur bei älteren Patienten, sondern auch bei Betroffenen mit Manifestationsalter zwischen dem 35. und 65. Lebensjahr die häufigste Ursache einer Demenz [18, 20, 21, 35]. Inner- 


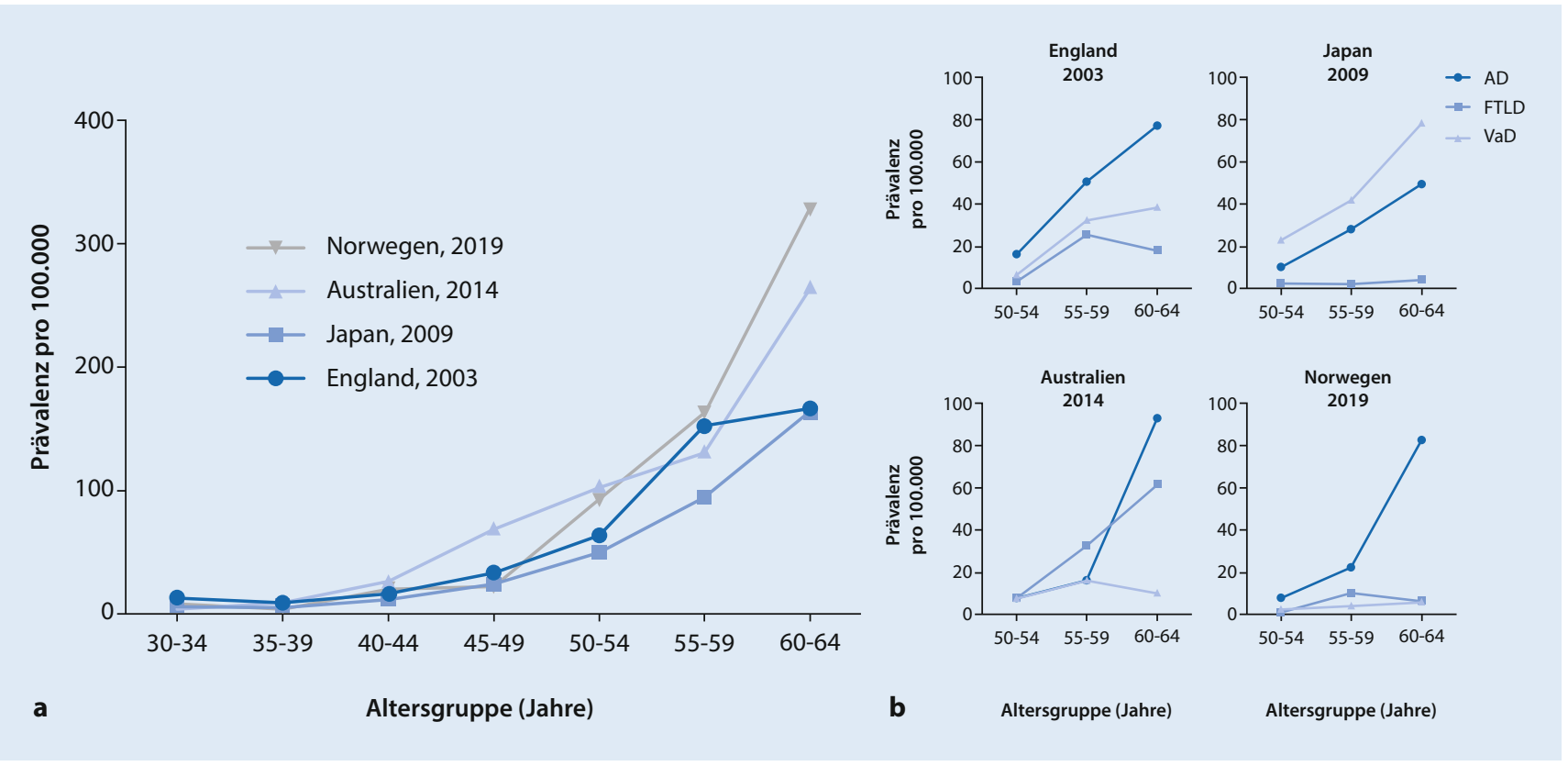

Abb. $1 \Delta$ a Prävalenz aller früh beginnenden Demenzen in vierverschiedenen Registerstudien [14, 16, 20,35].b Prävalenz der häufigsten Ursachen einer Demenz im Alter von 50 bis 64 Jahren. AD Alzheimer-Krankheit, FTLD frontotemporale Lobärdegeneration, $V a D$ vaskuläre Demenz

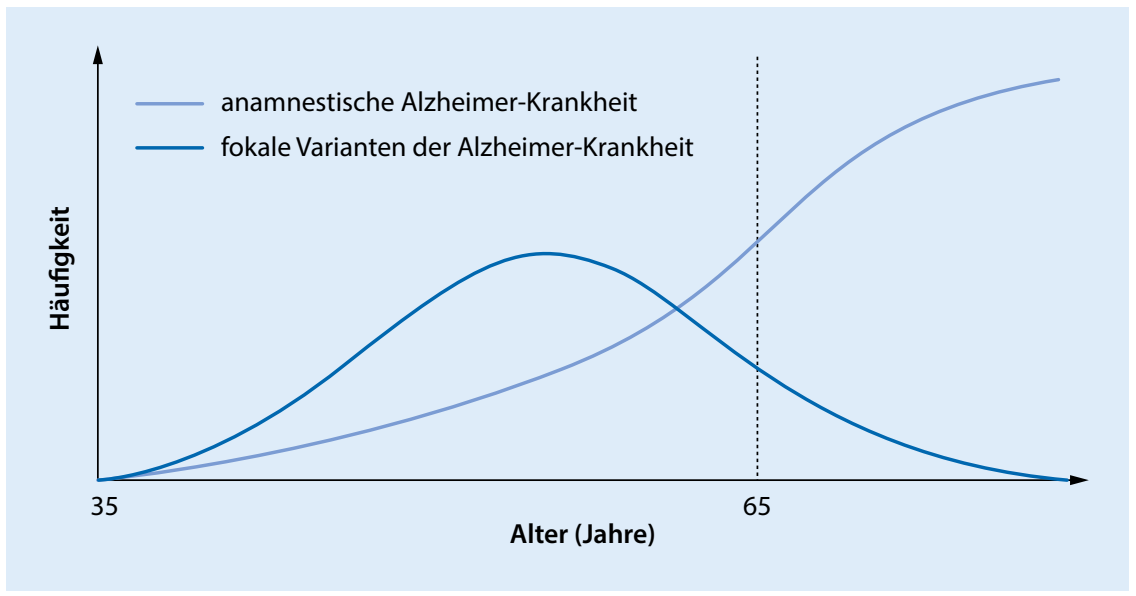

Abb. 2 \ Abhängigkeit der klinischen Präsentation der Alzheimer-Krankheit vom Erkrankungsalter

halb dieser Gruppe stellt die AlzheimerKrankheit die häufigste Ursache dar, gefolgt von der frontotemporalen Lobärdegeneration (FTLD) (• Abb. 1b). Die Lewy-Körperchen-Demenz und Parkinson-Demenz, die bei älteren Patienten die zweithäufigste Ursache einer Demenz darstellen, sind bei Patienten vor dem 65. Lebensjahr hingegen seltener. Die vaskuläre Demenz wird formal nicht zu den sekundären Demenzen gezählt. Ihre Häufigkeit bzw. ihr Anteil an den Ursachen früh beginnender Demenzen unterliegt möglicherweise starken geo- graphischen Schwankungen und war in einer japanischen Studie deutlich größer als in den europäischen/australischen Studien (• Abb. 1b; [14, 16, 20, 35]).

Bei Patienten mit sehr früher klinischer Manifestation, d.h. vor dem 35. Lebensjahr, fallen die primär neurodegenerativen Erkrankungen proportional kaum ins Gewicht, stattdessen finden sich prozentual deutlich häufiger sekundäre Demenzen, also neurokognitive Störungen z.B. infolge metabolischer, genetischer oder inflammatorischer Erkrankungen [18]. Ab dem
35. Lebensjahr gleichen sich die unterschiedlichen Ursachen und deren relative Häufigkeit zwischen Demenzen bei jüngeren ( $<65$ Jahre) und älteren ( $\geq 65$ Jahre) Menschen zunehmend an $[18,31]$. Umgekehrt gilt: Je früher das Manifestationsalter einer Demenz, desto eher handelt es sich um eine sekundäre Demenz [18, 31]. Sekundäre Demenzen sind bei jungen Menschen nicht nur prozentual deutlich häufiger als bei älteren Menschen, sondern das Spektrum der Differenzialdiagnosen ist auch wesentlich breiter (• Tab. 1; [10, 31]).

\section{Alzheimer-Krankheit}

Die Alzheimer-Krankheit manifestiert sich in unterschiedlichen klinischen Syndromen, welche alle durch die gleiche zugrunde liegende Neuropathologie gekennzeichnet sind: extrazelluläre Plaques aus Ablagerungen des Peptids Amyloid- $\beta$ $(\mathrm{A} \beta)$, intrazelluläre Ablagerungen des mikrotubuliassoziierten Proteins Tau und Neuroinflammation [34].

Bei der typischen Form der Alzheimer-Demenz, die sich in etwa $95 \%$ der Fälle jenseits des 65. Lebensjahres manifestiert [1], stehen initial mnestische Störungen im Vordergrund, genauer ei- 
ne Störung der Lern- und Merkfähigkeit, ohne oder nur mit geringem Profit durch Abrufhilfen. Im Gegensatz dazu, manifestiert sich die Krankheit bei jüngeren Patienten in etwa 20-65\% der Fälle in Form atypischer, fokaler Varianten (• Abb. 2; [23]): Die zwei häufigsten Formen sind die posteriore kortikale Atrophie (PCA) und die logopenische Variante der primär progressiven Aphasie (PPA). Seltener ist die frontale Variante der Alzheimer-Krankheit [34].

Bei der PCA kommt es zu vorwiegend visuellen Symptomen trotz intakter primärer visueller Verarbeitung, d.h. das Sehen ist unbeeinträchtigt, jedoch ist die Interpretation der Seheindrücke gestört [7]. Im Vordergrund steht somit eine komplexe visuell-perzeptive kognitive Störung mit Simultanagnosie, okulärer Apraxie, optischer Ataxie und visueller Agnosie [7].

Bei der logopenischen Variante der primär progressiven Aphasie (lpPPA) handelt es sich um eine zunächst isoliert auftretende Sprachstörung, charakterisiert durch Wortfindungsstörungen, eine reduzierte Sprachproduktion bei relativ gut erhaltener Phonologie und Syntax sowie ausgeprägten Störungen des verbalen Kurzzeitgedächtnisses, welches sich insbesondere durch fehlerhaftes Nachsprechen mehrsilbiger Wörter manifestiert [13, 24].

Die frontale Variante der AlzheimerKrankheit (auch als dysexekutive oder behaviorale Variante bezeichnet) weist klinisch zahlreiche Überschneidungen mit der behavioralen frontotemporalen Demenz auf: Sie ist durch eine frühzeitige Apathie und verminderte Initiierung produktiven Verhaltens (Apathie) gekennzeichnet, aber auch durch Disinhibition und Impulsivität [26].

\section{Frontotemporale Lobär- degeneration}

Die frontotemporale Lobärdegeneration (FTLD) ist ein spezifischer neuropathologischer Begriff für primär neurodegenerative Erkrankungen mit Atrophie frontaler und/oder temporaler Strukturen sowie histologisch nachweisbaren, intrazellulären Ablagerungen aberranter Formen der Proteine Tau, TDP-43

Nervenarzt 2020 $91: 936-945$ https://doi.org/10.1007/s00115-020-00967-0

(C) Der/die Autor(en) 2020

\section{Pawlowski $\cdot$ A. Johnen $\cdot$ T. Duning}

\section{Früh beginnende Demenzen}

\section{Zusammenfassung}

Hintergrund. Aufgrund des demographischen Wandels sind Demenzen ein häufiger und dramatisch zunehmender Grund für ärztliche Vorstellungen. In etwa $8 \%$ der Fälle treten sie bereits vor dem 65. Lebensjahr auf. Gerade bei jüngeren Patienten sind die psychosozialen und ökonomischen Folgen oft gravierend. Die Behandler stehen vor großen diagnostischen Herausforderungen. Eine rasche Diagnose ist für das Patientenmanagement von zentraler Bedeutung.

Ziel der Arbeit/Fragestellung. Dieser Übersichtsartikel stellt die Besonderheiten der Demenzen bei jüngeren Menschen sowie die wichtigsten zugrunde liegenden Krankheitsbilder vor und vermittelt ein strukturiertes klinisch-diagnostisches Vorgehen.

Methoden. Narrativer Review. Die Literatursuche wurde in PubMed durchgeführt. Ergebnisse. Das differenzialdiagnostische Spektrum von Demenzen bei jüngeren Menschen vor dem 65. Lebensjahr ist sehr breit. Die häufigsten Ursachen stellen die

\section{Young onset dementia}

\section{Abstract}

Background. Due to the demographic change dementia is a common and dramatically increasing reason for medical presentations. In approximately $8 \%$ of cases dementia occurs before the age of 65 years. The psychosocial and economic consequences are often severe, particularly in younger patients. Clinicians face major diagnostic challenges. A rapid diagnosis is crucial for patient counselling and management.

Objective. This review article presents the special features of dementia in younger people, the most important underlying diseases and a rational clinical diagnostic approach.

Methods. Narrative review. The literature search was carried out in PubMed.

Results. The differential diagnostic spectrum of dementia in younger people under the age of 65 years is very broad. The most common causes are Alzheimer's disease
Alzheimer-Krankheit mit typischen oder atypischen klinischen Präsentationen sowie die frontotemporale Lobärdegeneration dar. Je jünger das Erkrankungsalter, desto höher ist der Anteil an behandelbaren und potenziell reversiblen Ursachen eines demenziellen Syndroms.

Diskussion. Die Diagnostik primär neurodegenerativer Erkrankungen hat sich zunehmend verbessert, insbesondere unter Berücksichtigung einer stetig steigenden Zahl an klinischen, molekularen und bildgebenden Biomarkern. Dennoch muss die Diagnostik der Demenzen mit frühem Erkrankungsbeginn hypothesengeleitet erfolgen, d.h. nach einer präzisen klinisch-syndromalen Zuordnung der Symptome. So können unnötige und belastende Untersuchungen vermieden werden.

\section{Schlüsselwörter}

Präsenile Demenzen · Sekundäre Demenzen . Frontotemporale Lobärdegeneration . Alzheimer-Krankheit · Vaskuläre Demenz with typical or atypical clinical presentations and frontotemporal lobar degeneration. The younger the age of onset, the higher the proportion of treatable and potentially reversible causes of dementia.

Conclusion. The diagnostics of primary neurodegenerative diseases have continuously improved, especially due to the availability of an increasing number of clinical, molecular and imaging biomarkers. Nevertheless, in order to avoid unnecessary and burdensome examinations, the diagnostic work-up of young onset dementia must be hypothesisdriven, i.e. following a precise clinical syndromic classification of the symptoms.

\section{Keywords}

Early-onset dementia - Secondary dementia . Frontotemporal lobar degeneration . Alzheimer disease - Vascular dementia 


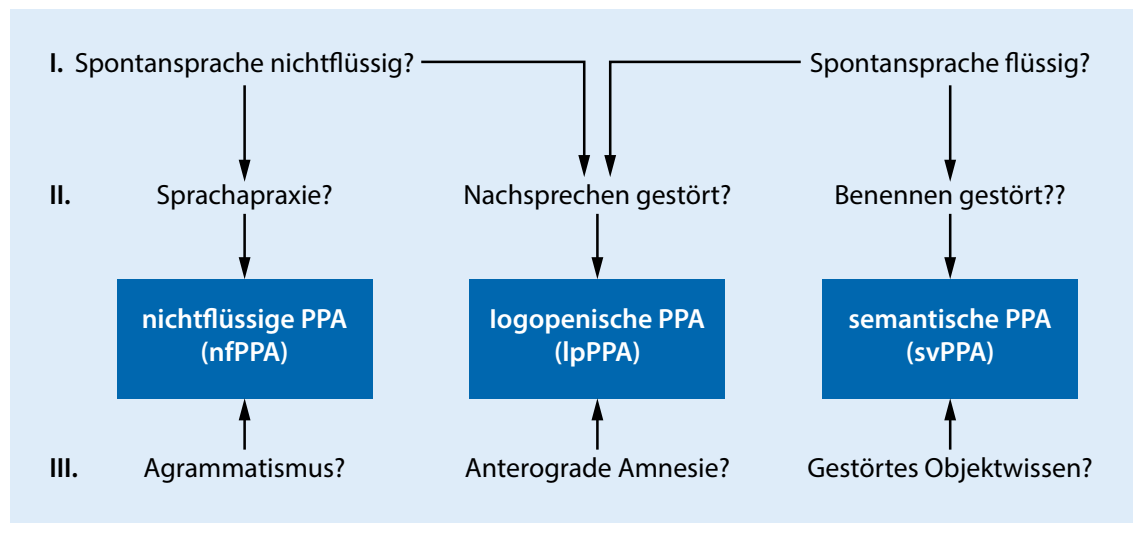

Abb. 3 A Algorithmus zur klinischen Bedside-Differenzierung der drei primär progressiven Aphasien $(P P A)$ im klinischen Alltag

oder FUS [2]. Das klinische Korrelat der FTLD ist in den meisten Fällen eine frontotemporale Demenz (FTD): Hierzu zählen die Verhaltensvariante der FTD (bvFTD) und die Sprachvarianten, also die verschiedenen Subtypen der PPA. Das Erkrankungsalter von FTD-Patienten liegt meist zwischen dem 45. und dem 65. Lebensjahr [2].

Die behaviorale Variante der FTD (bvFTD) stellt die häufigste klinische Präsentation einer FTLD dar. Die Erstsymptome sind häufig subtil, die Patienten zeigen fast immer eine Anosognosie für die leitsymptomatischen Verhaltensänderungen, welche von Angehörigen oftmals zunächst als "midlife crisis“ fehlinterpretiert werden. Diese Präsentation resultiert aus den zentralen bvFTDSymptomen, die auch die Grundlage der Diagnosekriterien nach Rascovsky bilden: sog. „Plussymptome“ wie soziale Disinhibition, perseverierende, stereotype oder zwanghaft, ritualisierte Verhaltensmuster oder Veränderungen im Essverhalten (insbesondere ein gesteigerter Konsum von Süßigkeiten). Andererseits kommt es häufig bei denselben Patienten auch zu Minussymptomen wie Apathie, Antriebsarmut oder Verlust von Empathie [22]. Neben den Verhaltenssymptomen finden sich markante Störungen exekutiver Funktionen trotz relativ intakter Gedächtnisleistungen und visuell-räumlicher kognitiver Funktionen [28]. Die Diagnosekriterien einer "wahrscheinlichen bvFTD“ fordern zudem das Vorhandensein einer Alltagsrelevanz der genannten Symptome, eine Verschlechterung in Bezug auf die drei PPA-Varianten häufig klinisch markant differenzieren (• Abb. 3).

\section{Vaskuläre Demenz}

Die vaskuläre Demenz wird in einigen epidemiologischen Studien als häufige Ursache einer Demenz bei jüngeren Menschen angegeben $[14,16]$. In diesen Studien verbirgt sich hinter dem Begriff der vaskulären Demenz jedoch eine sehr heterogene Gruppe ätiologisch unterschiedlicher Erkrankungen. Im engeren Sinn versteht man unter einer vaskulären Demenz ein subkortikales demenzielles Syndrom infolge einer zerebralen Mikroangiopathie [30]. Klinisch imponiert eine psychomotorische Verlangsamung mit einer Störung des Abrufens bereits erlernter Gedächtnisinhalte sowie reduzierter Verarbeitungsgeschwindigkeit und Aufmerksamkeit. Die Patienten wirken im Gegensatz zu AlzheimerPatienten im klinischen Alltag umständlich, leicht ablenkbar und wenig fokussiert. Die zerebrale Mikroangiopathie ist vor dem 65 Lebensjahr sehr selten und deshalb auch ein seltener Grund für eine früh beginnende Demenz. Im weiteren Sinn werden jedoch auch strategisch lokalisierte embolische Infarkte (z. B. in Thalamus, Fornix oder Hippokampus), die zerebrale Amyloidangiopathie, primäre und sekundäre zerebrale Vaskulitiden sowie genetische Erkrankungen wie die zerebrale autosomal-dominante Arteriopathie mit subkortikalen Infarkten und Leukenzephalopathie (CADASIL) zur vaskulären Demenz gezählt. Gemäß der aktuellen S3-Leitlinie stellt für die Diagnose einer vaskulären Demenz das Vorhandensein vaskulärer zerebraler Läsionen und ein zeitlicher Zusammenhang mit dazu passenden kognitiven Funktionsstörungen das dominante diagnostische Kriterium dar [9]. Die oben genannten, heterogenen Ursachen der vaskulären Demenzen sind für sich allein genommen selten, machen in ihrer Summe mit etwa $18 \%$ jedoch einen relativ hohen Anteil der früh beginnenden Demenzen aus [14, 31]. 


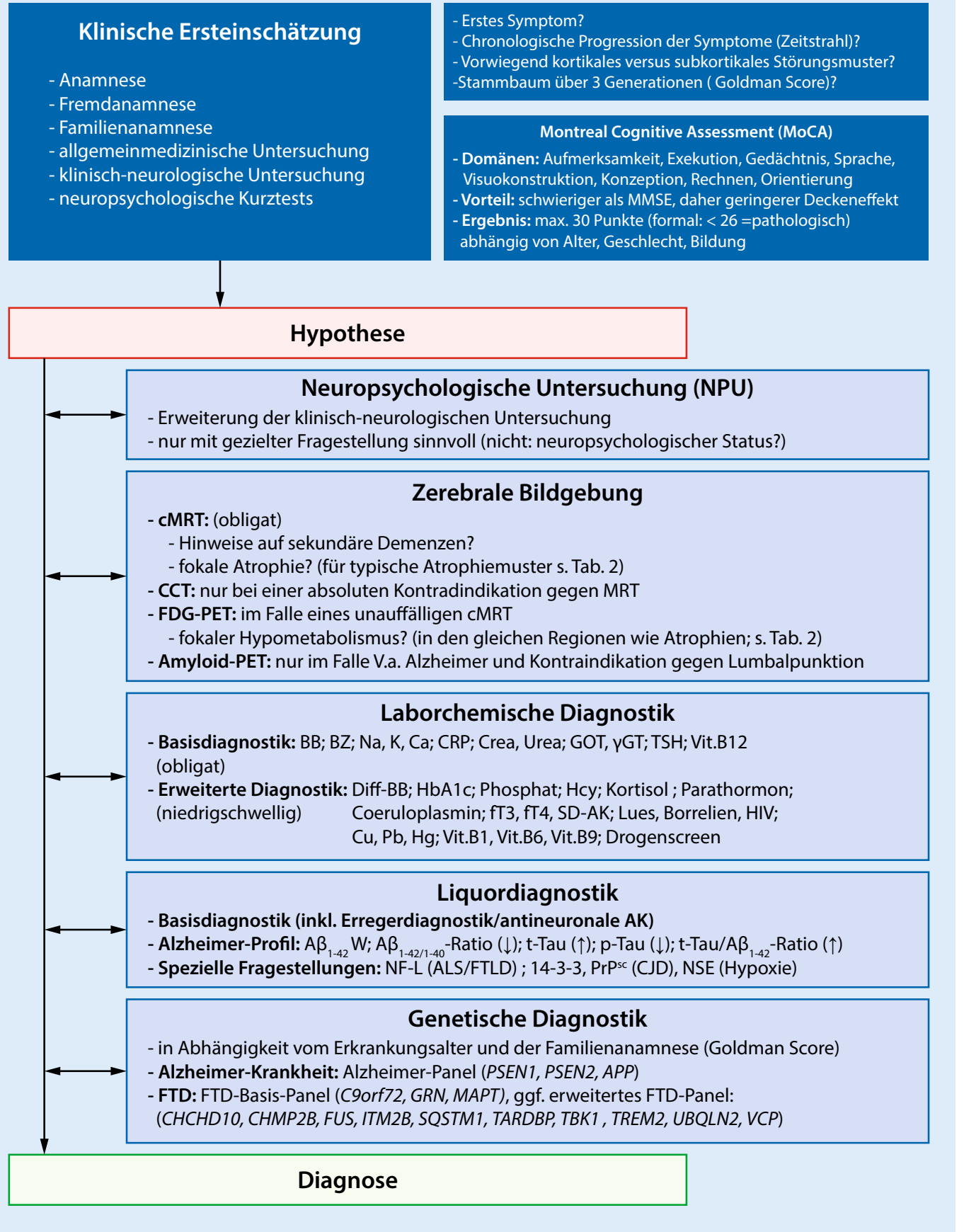

\begin{abstract}
Abb. $4 \triangleleft$ Algorithmus zum diagnostischen Vorgehen bei Patienten mit früh beginnender Demenz. MMSE Mini-Mental State Examination, $C M R T$ kraniale Magnetresonanztomographie, CCT kraniale Computertomographie, FDG-PET Fluordeoxyglukose-Positronenemissionstomographie, $B B$ Blutbild, $B Z$ Blutzucker, CRPC-reaktives Protein, Crea Kreatinin, Hcy Homozystein, fT3/4 freie Schilddrüsenhormone, SD-AK Schilddrüsenantikörper (TRAK, TPO, TG), Cu Kupfer, Pb Blei, $\mathrm{Hg}$ Quecksilber, NF-L Neurofilament-Leichtketten, CJD Creutzfeld-JakobKrankheit, NSE neuronenspezifische Enolase
\end{abstract}

\section{Sekundäre Demenzen}

Unter dem Oberbegriff ,sekundäre Demenzen" werden alle demenziellen Syndrome zusammengefasst, die nicht Folge einer primär neurodegenerativen Erkrankung sind und nicht zu den vaskulären Demenzen zählen. Die kognitiven Störungen können dabei Folge einer sekundären zerebralen Funktionsstörung oder einer sekundären Neurodege- neration sein. Sekundäre Demenzen sind bei jungen Menschen prozentual deutlich häufiger als bei älteren Menschen. Sie machen in ihrer Gesamtheit bei Patienten $<65$ Jahre knapp ein Drittel aller Fälle aus, bei Patienten $<35$ Jahre stellen sie sogar die häufigste Ursache einer Demenz dar $[14,18,31]$. Das Feld der Differenzialdiagnosen ist sehr breit: Erkrankungen, die sich mit einem sekundären demenziellen Syndrom manifestieren, umfassen verschiedene Infektionskrankheiten, autoimmunvermittelte Erkrankungen, metabolische und hereditäre Erkrankungen, ethyltoxische oder traumatische Hirnschäden. Einige der sekundären Demenzen sind sehr gut behandelbar und sollten daher nicht übersehen werden. Aus diagnostischer Sicht ist in diesem Zusammenhang das Konzept der Demenz-Plussyndrome von praktischer Bedeutung. Bei vielen der genannten Erkrankungen 
Tab. 1 Auswahl der wichtigsten Differenzialdiagnosen früh beginnender Demenzen

\begin{tabular}{|c|c|c|c|}
\hline Gruppe & Diagnose & \multicolumn{2}{|c|}{ „Red flags" (Symptome/Tests) } \\
\hline \multirow{4}{*}{$\begin{array}{l}\text { Primär } \\
\text { neurodegenerative } \\
\text { Erkrankungen }\end{array}$} & Alzheimer-Krankheit & \multicolumn{2}{|c|}{ Anterograde mnestische Defizite } \\
\hline & FTLD (inkl. PSP, CBD), ALS & \multicolumn{2}{|c|}{ Paresen, Atrophien, PBZ } \\
\hline & PDD, LBD, MSA & \multicolumn{2}{|c|}{ Parkinson-Symptome, zerebelläre Symptome, subkortikales Störungsmuster } \\
\hline & Creutzfeld-Jakob-Krankheit (sCJD) & \multicolumn{2}{|c|}{ Myoklonus, Ataxie, PBZ, Mutismus } \\
\hline \multirow[t]{3}{*}{ Vaskuläre Demenz } & Zerebrale Mikroangiopathie & \multicolumn{2}{|c|}{ Subkortikales Störungsmuster } \\
\hline & Multiinfarktsyndrom & \multicolumn{2}{|c|}{ MRT: strategische Infarkte } \\
\hline & Zerebrale Vaskulitis & \multicolumn{2}{|c|}{$\begin{array}{l}\text { Hirninfarkte; Labor: entz. Liquor } \\
\text { MRT: Gefäßkaliberirregularitäten }\end{array}$} \\
\hline Hydrozephalus & Normaldruckhydrozephalus (NPH) & \multicolumn{2}{|c|}{ Inkontinenz, Gangstörung, subkortikales Störungsmuster; MRT: Evans-Index $>0,3$} \\
\hline Trauma & $\begin{array}{l}\text { Chronisch-traumatische Enzephalo- } \\
\text { pathie }\end{array}$ & \multicolumn{2}{|c|}{ Positive Trauma-/Sportanamnese; MRT: zerebrale Mikroscherverletzungen } \\
\hline Ernährung/ & Alkoholkrankheit & \multicolumn{2}{|c|}{ Desorientiertheit, Augenmuskelparesen, Ataxie } \\
\hline $\begin{array}{l}\text { Toxische } \\
\text { Suhstanzen }\end{array}$ & Vitaminmangel & \multicolumn{2}{|c|}{ Vitamin B1, B3, B6, B9, B12, D } \\
\hline & Dauerhafte Pharmakotherapie & \multicolumn{2}{|c|}{ Trizyklika, Lithium, Valproat, Antipsychotika } \\
\hline Metabolisch/ & Hepatopathie & \multicolumn{2}{|c|}{ Flapping-Tremor, Labor: Leberwerte (v. a. $\left.\mathrm{NH}_{3}\right)$} \\
\hline & Nephropathie & \multicolumn{2}{|c|}{ Labor: Nierenretentionsparameter (v. a. Urea) } \\
\hline & Schilddrüsenfunktionsstörung & \multicolumn{2}{|c|}{ Hypothyreose, seltener Hyperthyreose } \\
\hline $\begin{array}{l}\text { Autoimmunvermit- } \\
\text { telte bzw. }\end{array}$ & Multiple Sklerose (MS) & \multicolumn{2}{|c|}{$\begin{array}{l}\text { Fokal-neurologische Defizite } \\
\text { Labor: entz. Liquor; MRT: MS-Läsionen }\end{array}$} \\
\hline $\begin{array}{l}\text { chronisch-ent- } \\
\text { zündliche }\end{array}$ & Neuromyelitis optica (NMOSD) & \multicolumn{2}{|c|}{$\begin{array}{l}\text { Optikusneuritis, transverse Myelitis } \\
\text { Labor: entz. Liquor; Anti-AQP4, MOG-AK }\end{array}$} \\
\hline & $\begin{array}{l}\text { Limbische Enzephalitis } \\
\text { - Autoimmunenzephalitis } \\
\text { - Paraneoplastische Enzephalitis }\end{array}$ & \multicolumn{2}{|c|}{$\begin{array}{l}\text { Epileptische Anfälle, psychotische Symptome } \\
\text { Labor: entz. Liquor; Anti-LGI1, CASPR2, GAD, AMPAR, GABA }{ }_{B} R \text {, Hu, Ma2-AK i.S./L. } \\
\text { MRT: temporomesiale T2-Hyperintensität } \\
\text { EEG: bitemporale epilepsietypische Potenziale }\end{array}$} \\
\hline & SREAT (Hashimoto-Enzephalitis) & \multicolumn{2}{|c|}{ Epileptische Anfälle, Myoklonus, psychotische Symptome; Labor: Anti-TPO, TG-AK } \\
\hline & Sarkoidose & \multicolumn{2}{|c|}{$\begin{array}{l}\text { Mediastinale Lymphadenopathie, Arthralgie, Erythema nodosum; Liquor: Schrankenstö- } \\
\text { rung }\end{array}$} \\
\hline \multirow{5}{*}{$\begin{array}{l}\text { Infektionskrank- } \\
\text { heiten }\end{array}$} & HIV & \multicolumn{2}{|c|}{ Opportunistische Infektionen, PNP } \\
\hline & Neurolues & \multicolumn{2}{|c|}{ Tabes dorsalis, oft Koinfektion mit HIV } \\
\hline & Neuroborreliose & \multicolumn{2}{|c|}{ Erythema migrans, Bannwarth-Syndrom } \\
\hline & Whipple-Krankheit & \multicolumn{2}{|c|}{ Diarrhö, Malabsorption, Parkinson-Symptome } \\
\hline & Herpesenzephalitis & \multicolumn{2}{|c|}{ Epileptische Anfälle, Aphasie, Vigilanzminderung } \\
\hline Leukodystrophien & Metachromatische Leukodystrophie & $A R S A ; \mathrm{AR}$ & Augen-, Haut-Symptome, epileptische \\
\hline & Adrenoleukodystrophie & $A B C D 1 ; \mathrm{XR}$ & \\
\hline & Krabbe-Krankheit & GALC; XR & Kleinhirnsymptome, Bronze-Diabetes \\
\hline & Alexander-Krankheit & GFAP; AD & MRT: bilateral symmetrisch, konfluierende \\
\hline & CADASIL & NOTCH3; AD & Leukenzephalopathie \\
\hline & Zerebrotendinöse Xanthomatose & CYP27A1; AR & \\
\hline & Adulte auto.-dom. Leukodystrophie & $L M N B 1 ; A D$ & \\
\hline & $\begin{array}{l}\text { Adulte Polyglucosankörpererkran- } \\
\text { kung }\end{array}$ & GBE1; AR & \\
\hline & $\begin{array}{l}\text { Adulte Leukoenzephalopathie mit } \\
\text { axonalen Sphäroiden }\end{array}$ & CSF1R; AD & \\
\hline & Fragile-X-Tremor-Ataxie-Syndrom & FMR1; XD & \\
\hline
\end{tabular}




\begin{tabular}{|c|c|c|c|}
\hline Gruppe & Diagnose & „Red flags" (Symptome/Tests) & \\
\hline \multirow{9}{*}{$\begin{array}{l}\text { Andere hereditäre } \\
\text { Erkrankungen }\end{array}$} & Huntington-Krankheit & HTT; AD & Choreatiforme Störung \\
\hline & Niemann-Pick-Krankheit Typ C & $N P C 1 ; A R$ & $\begin{array}{l}\text { Ataxie, Slow-Blicksakkaden, Kataplexie, } \\
\text { Splenomegalie }\end{array}$ \\
\hline & Fabry-Krankheit & $G L A ; \mathrm{XR}$ & Parästhesien, Angiokeratome, Hirninfarkte \\
\hline & Wilson-Krankheit & $A T P 7 B ; \mathrm{XR}$ & $\begin{array}{l}\text { Extrapyramidalmotorische Störungen, } \\
\text { Hepatopathie }\end{array}$ \\
\hline & Mitochondriopathien & \multicolumn{2}{|l|}{ Myopathie, epileptische Anfälle, PNP } \\
\hline & Spinozerebelläre Ataxie & \multicolumn{2}{|l|}{ Zerebelläre Symptome } \\
\hline & Hereditäre spastische Paralyse & \multicolumn{2}{|l|}{ Paraparese, PBZ, Inkontinenz } \\
\hline & $\begin{array}{l}\text { Neurodegeneration mit Eisenspei- } \\
\text { cherung }\end{array}$ & \multicolumn{2}{|l|}{$\begin{array}{l}\text { Extrapyramidalmotorische Störungen } \\
\text { MRT: erhöhte Eisenspiegel in Basalganglien }\end{array}$} \\
\hline & Neuroakanthozytose & \multicolumn{2}{|l|}{ Dyskinesien; Labor: Akanthozyten } \\
\hline \multicolumn{4}{|c|}{ 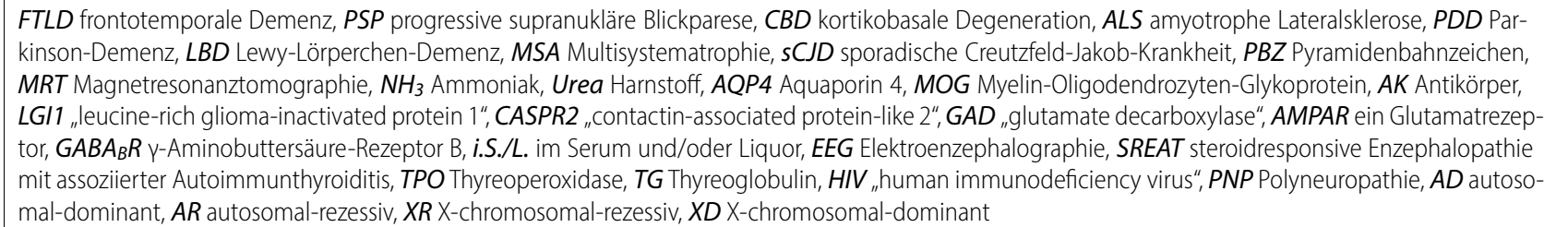 } \\
\hline
\end{tabular}

handelt es sich um Systemerkrankungen, bei denen das demenzielle Syndrom nicht die einzige Krankheitsmanifestation darstellt, sondern lediglich eines von mehreren Symptomen. Bei den Plussymptomen kann es sich sowohl um andere neurologische (z. B. motorische) Symptome oder um Symptome infolge einer Beteiligung anderer Organsysteme handeln [31].

\section{Diagnostisches Vorgehen}

Die korrekte Diagnose bei jüngeren $\mathrm{Pa}$ tienten mit einer Demenz erfordert ein strukturiertes diagnostisches Vorgehen. In Abwesenheit spezifischer Biomarker für den Großteil der möglichen zugrunde liegenden Erkrankungen basiert die Diagnose primär auf den klinischen Symptomen. Die Diagnostik bei jungen Patienten mit Demenz sollte daher hypothesengeleitet erfolgen, d.h. nach einer präzisen klinisch-syndromalen Zuordnung der Symptome (• Abb. 4).

\section{Klinischer Eindruck}

Die Erstvorstellung erfolgt in den meisten Fällen zur Abklärung subjektiv empfundener kognitiver Funktionsstörungen, die entweder der betroffenen Person selbst oder Angehörigen aufgefallen sind. Von größter differenzialdiagnostischer Bedeutung ist die Identifizierung des zuerst aufgetretenen Krankheitssymptoms sowie dessen zeitlicher Verlauf. Aufgrund der Art der vorhandenen kognitiven Störungen oder dem Vorliegen einer Anosognosie ist die Anamnese der betroffenen Person in vielen Fällen nicht ausreichend und eine ergänzende strukturierte Fremdanamnese unabdingbar; gleiches gilt für die Familienanamnese.

Auch wenn die Einteilung aus neuropathologischer und neuropsychologischer Sicht stark vereinfacht erscheint, ist eine initiale Einteilung der kognitiven Funktionsstörung in kortikale „Werkzeugstörungen“ oder subkortikale kognitive Störungen zur Planung des diagnostischen Vorgehens empfehlenswert. $\mathrm{Zu}$ den kortikalen neurokognitiven Funktionen zählen Gedächtnis, Sprache, Praxie und Visuokonstruktion. Störungen in diesen Domänen sind typische Symptome der AlzheimerKrankheit oder der FTLD. Den kortikalen Symptomen gegenüberzustellen sind subkortikale Störungen [15]. Hierzu zählt in erster Linie die psychomotorische Verlangsamung. Das Abrufen bereits erlernter Gedächtnisinhalte ist erschwert, Verarbeitungsgeschwindigkeit und Aufmerksamkeitsleistungen sind reduziert. Patienten mit subkortikalen Störungen wirken im Kontakt unkonzentriert, umständlich, ohne Fokus auf das Wesentliche, fahrig und leicht ablenkbar. Typisches Beispiel für eine subkortikale Demenz ist die zerebrale Mikroangiopathie, aber auch viele sekundäre Demenzen zeigen ein subkortikales Minderleistungsprofil.

Bei jedem Patienten mit Demenzverdacht sollte bereits bei der Erstdiagnose eine standardisierte Quantifizierung der kognitiven Leistungseinbußen erfolgen. Es stehen verschiedene zeitökonomische Tests zur Verfügung, die jeweils mit Vor- und Nachteilen behaftet sind. In der Ersteinschätzung ist ein Multidomänen-Screeningtest sinnvoll [5], z.B. der Mini-Mental State Test (MMST; [11]) oder das Montreal Cognitive Assessment (MoCA; [25]). Der MMST ist weltweit am meisten verbreitet [11]. Für die Frühdiagnose besteht aber ein erheblicher Deckeneffekt (zu einfache Items). Zudem werden v. a. subkortikale Domänen oder Exekutivfunktionen nicht ausreichend erfasst [5]. Das MoCA hat ein ähnliches Design und die gleiche maximal erreichbare Gesamtpunktzahl (30 Punkte) wie der MMST. Es wurde jedoch primär als spezifisches Screeninginstrument zur Detektion leichtgradiger kognitiver Störungen entwickelt, die einzelnen Aufgaben sind schwieriger und die Auswahl kognitiver Domänen breiter gewählt. Dadurch fällt der Deckeneffekt des Testes deutlich geringer aus und das Spektrum der detektierbaren 
Tab. 2 Typische zerebrale Atrophiemuster der primär neurodegnerativen Erkrankungen

\section{Klinisches Syndrom}

Amnestisches Syndrom

Posteriore kortikale Atrophie

Verhaltensauffälligkeiten

Nichtflüssige PPA (nfPPA)

Semantische PPA (svPPA)

Logopenische PPA (IpPPA)

\section{Pathologie}

$A D>>$ FTLD

$A D>>F T L D$

FTLD $>>A D$

FTLD $>$ AD

FTLD

$A D>$ FTLD

\section{MRT-Atrophie}

Medial temporal

Okzipital/parietal/temporal

Frontal/anterior temporal

Posterior frontoinsular (links)

Anterior temporal (links $>$ rechts)

Temporal/parietal (links)
Differenzialdiagnosen ist insbesondere bei jüngeren Patienten breiter [5]. Ein Ergebnis von 26 oder mehr Punkten gilt als normal [25]. Zahlreiche Validierungsstudien zeigen jedoch, dass dieser Cut-off-Wert in vielen Fällen zu streng gewählt wurde. Eine rezente Validierungsstudie der offiziellen deutschen Übersetzung des MoCA demonstrierte eine Spannweite des Cut-off-Wertes von 17 bis 26 Punkten in Abhängigkeit von Geschlecht, Alter und Bildung, die anstelle des starren 26-Punkte-Wertes genutzt werden sollte [33].

Neben der Anamnese ist eine ausführliche medizinische und klinischneurologische Untersuchung erforderlich, um systemische oder neurologische Demenz-Plussymptome als Hinweise auf bestimmte Ursachen einer sekundären Demenz nicht zu übersehen. - Tab. 1 zeigt eine Übersicht der wichtigsten Differenzialdiagnosen früh beginnender Demenzen mit v. a. internistischen oder klinisch-neurologischen „red flags" bzw. typischen Ergebnissen der laborchemischen oder bildgebenden Diagnostik. Es ist von entscheidender Bedeutung, potenziell reversible und behandelbare Ursachen nicht zu übersehen. Diese reichen vom Normaldruckhydrozephalus, welcher bereits ab dem 40 . Lebensjahr auftreten kann, über die Alkoholkrankheit und Hypovitaminosen bis hin zu den autoimmunvermittelten oder infektiösbedingten Enzephalitiden sowie den hereditären Stoffwechselstörungen. Gerade im vergangenen Jahrzehnt haben die Autoimmunenzephalitiden zunehmend an Bedeutung gewonnen. Durch die Entdeckung spezifischer Autoantikörper gegen neuronale Antigene im ZNS gelang die Klassifizierung spezifischer Krankheitsentitäten. Im Erwachsenenalter neu auftretende kognitive Defizite sind eine typische Präsentation der limbischen Enzephalitiden, meist charakterisiert durch episodische Gedächtnisstörungen, epileptische Anfälle, bilaterale T2/ FLAIR-Hyperintensitäten temporomesialer Strukturen im MRT, bitemporale epilepsietypische Potenziale im EEG und den laborchemischen Nachweis spezifischer Autoantikörper im Liquor oder v. a. im Serum (『Tab. 1).

\section{Ausführliche neuropsychologische Testung}

Eine ausführliche neuropsychologische Untersuchung (NPU) ist ein integraler Bestandteil des diagnostischen Assessments und sollte als Erweiterung der klinischen Untersuchung zur Einschätzung höherer kognitiver Funktionen eingesetzt werden [36]. Im Gegensatz zu neuropsychologischen Kurztests liefert eine NPU qualitative und quantitative Information über die Funktion der wesentlichen kognitiven Domänen (komplexe Aufmerksamkeit, exekutive Funktionen, Lernen und Gedächtnis, Sprache, perzeptuell-motorische Fähigkeiten und soziale Kognition) sowie deren Einfluss auf mögliche individuelle Einschränkungen im Alltag der Patienten [36]. Den verschiedenen kognitiven Funktionen liegt die Integrität ausgedehnter, neuronaler Netzwerke zugrunde, weshalb bestimmte Konstellationen kognitiver Störungen auf eine Pathologie in spezifischen Hirnregionen hinweisen können. Es sollte jedoch nicht bei allen Patienten niedrigschwellig eine wenig fokussierte neuropsychologische Testung erfolgen. Stattdessen ist eine spezifische, hypothesengeleitete Fragestellung, basierend auf dem klinischen Eindruck unabdingbar, um die Effektivität der neuropsychologischen Untersuchung zu erhöhen [17].

\section{Zerebrale Bildgebung}

Jeder junge Patient mit neu aufgetretenen kognitiven Störungen muss eine zerebrale Bildgebung erhalten. Die Magnetresonanztomographie (MRT) ist der Computertomographie (CT) aufgrund der größeren Genauigkeit bei jung Erkrankten vorzuziehen. Primär geht es dabei um den Ausschluss symptomatischer Ursachen der Demenz (z.B. Tumoren, entzündliche oder vaskuläre Läsionen, siehe - Tab. 1). Über die Hälfte der sekundären oder reversiblen Demenzen mit frühem Manifestationsalter können mithilfe der strukturellen MRT diagnostiziert werden [6]. Bei Patienten mit vorwiegend kortikalen Symptomen stellt sich zudem die Frage nach spezifischen Atrophiemustern (•Tab.2). Eine Indikation für eine MRT zur routinemäßigen Verlaufskontrolle besteht im Regelfall nicht, kann bei atypischen Verläufen oder diagnostischer Unsicherheit aber sinnvoll sein.

Die strukturelle MRT ist in frühen Stadien primärer Demenzen oft wenig wegweisend. In Einzelfällen ist es deshalb sinnvoll, die strukturelle MRT um eine funktionelle Bildgebung zu ergänzen (z. B. Fluordesoxyglucose(FDG)Positronenemissionstomographie [PET] zum Nachweis eines regionalen Hypometabolismus, Dopamintransporter(DAT)Scan zum Nachweis eines striatalen Dopamindefizits [bei unklaren motorischen Symptomen] oder Amyloid-PET zum Nachweis regionaler $A \beta$-Ablagerungen). Eine funktionelle Bildgebung ist nicht indiziert, wenn sich im konventionellen MRT bereits ein klassisches Atrophiemuster zeigt.

\section{Laborchemische Untersuchungen und Liquordiagnostik}

Durch laborchemische Untersuchungen können potenziell reversible Ursachen einer Demenz diagnostiziert werden. Dabei sollte die Demenzbasisdiagnostik, welche für alle Patienten mit einer Demenz unabhängig vom Erkrankungsalter gilt, bei jungen Menschen niederschwellig um weiterführende Serum- bzw. Plasmauntersuchungen ergänzt werden (• Abb. 4; [9]). 


\section{Tab. 3 Modifizierter Goldman-Score}

\begin{tabular}{|c|c|}
\hline Score & Beschreibung \\
\hline $\begin{array}{l}\text { Score } 1 \\
\text { Autosomal-dominante Verer- } \\
\text { bung }\end{array}$ & $\begin{array}{l}\geq 3 \text { betroffene Verwandte in } 2 \text { Generationen, wobei eine der } 3 \\
\text { betroffenen Personen Verwandter } 1 \text {. Grades beider anderen ist }\end{array}$ \\
\hline $\begin{array}{l}\text { Score } 2 \\
\text { Familiäre Häufung }\end{array}$ & $\begin{array}{l}\geq 3 \text { betroffene Verwandte, ohne dass die Kriterien für Score } 1 \\
\text { erfüllt sind }\end{array}$ \\
\hline $\begin{array}{l}\text { Score } 3(a / b) \\
\text { Ein betroffener Verwandter } \\
\text { 1. Grades }\end{array}$ & $\begin{array}{l}\text { Manifestationsalter des betroffenen Verwandten: } \\
\text { a) }<65 \text { Jahre } \\
\text { b) } \geq 65 \text { Jahre }\end{array}$ \\
\hline $\begin{array}{l}\text { Score } 4 \\
\text { Keine relevante Familien- } \\
\text { anamnese }\end{array}$ & $\begin{array}{l}\text { Die Familienanamnese erfüllt nicht die Kriterien für Score } 1 \text { bis } 3 \\
\text { oder die Familienanamnese ist unbekannt }\end{array}$ \\
\hline
\end{tabular}

Eine Liquordiagnostik wird beijungen Menschen mit einer Demenz empfohlen [9]. Sie ist wichtig für die Identifizierung potenziell behandelbarer Infektionen oder autoimmunvermittelter Ursachen. Zudem erfolgt die Bestimmung der Konzentration spezifischer Proteine, die wichtige Hinweise auf das Vorhandensein bestimmter neurodegenerativer Erkrankungen liefern kann. Etablierte Biomarker der Alzheimer-Krankheit sind die verschiedenen Formen der Proteine Amyloid- $\beta(A \beta)$ und Tau. Von ersterem sollten die $A \beta_{1-42}$-Isoform sowie die $A \beta_{1-42} / A \beta_{1-40}$-Ratio bestimmt werden, von letzterem sowohl das Gesamtprotein (t-Tau) als auch die Threonin(T)-181oder T-231-phosphorylierte Form ( $\mathrm{p}$ Tau; [27]). Das typische Alzheimer-Liquorbiomarkerprofil, welches Bestandteil der Diagnosekriterien der AlzheimerKrankheit ist, umfasst eine Reduktion der Konzentration von $A \beta_{1-42}$, eine Reduktion der $A \beta_{1-42} / A \beta_{1-40}$-Ratio sowie eine Erhöhung von t-Tau und $\mathrm{p}$-Tau. Die Erhöhung von t-Tau für sich allein genommen ist ein molekularer Biomarker für den Untergang von Nervenzellen, ohne Spezifität für die zugrunde liegende Ursache. Ähnliches gilt für die Proteine 14-3-3, neuronenspezifische Enolase (NSE) und Neurofilament-Leichtketten (NF-L), die insbesondere bei Patienten mit Verdacht auf Creutzfeld-JakobKrankheit (14-3-3), posthypoxischen Hirnschäden (NSE) oder der amyotrophen Lateralsklerose (ALS) und der FTLD (NF-L) bestimmt werden und in Einzelfällen einen diagnostischen $\mathrm{Zu}$ satznutzen bieten können. Auch der Nachweis fehlgefalteten Prionproteins $\left(\mathrm{PrP}^{\mathrm{Sc}}\right)$ im Liquor bei Patienten mit spo- radischer Creutzfeld-Jakob-Krankheit ist möglich [32].

\section{Molekulargenetik}

In begründeten Verdachtsfällen, insbesondere bei jungen Menschen mit positiver Familienanamnese oder typischen Symptomkonstellationen, kann eine molekulargenetische Diagnostik zum Nachweis krankheitsverursachender Mutationen erfolgen. Monogenetisch verursachte Formen der Alzheimer-Krankheit sind jedoch selten: Weniger als $1 \%$ aller Patienten mit einer Alzheimer-Krankheit weisen eine autosomal-dominant vererbte Mutation in einem der drei häufigsten Gene PSEN1, PSEN2 und APP auf, die Penetranz bei vorhandener Mutation beträgt jedoch nahezu $100 \%$. Monogenetische autosomal-dominante Mutationen sind wahrscheinlich ursächlich in ca. $10-30 \%$ aller FTD-Fälle. Monogenetische Mutationen sind in $90 \%$ der Fälle in einem der drei Gene MAPT, GRN und C9orf72 lokalisiert, daneben existiert noch eine Vielzahl weiterer Gene, die zusammen jedoch nur knapp $5 \%$ der monogenetisch determinierten FTLD ausmachen (-Abb.4). Die Penetranz der FTLDverursachenden Genmutationen ist variabler.

Allgemein akzeptierte Algorithmen zur genetischen Testung bei Patienten mit einer primär neurodegenerativen Demenz liegen derzeit nicht vor [12]. Eine Testung kommt bei jungem Erkrankungsalter und mehreren betroffenen Familienangehörigen in Betracht. Bei der Erhebung der Familienanamnese sollte ein Stammbaum über drei Generationen erhoben werden. Mithilfe des modifizierten Goldman-Scores lässt sich das Ergebnis der Familienanamnese bzw. Stammbaumanalyse klassifizieren [3] und anhand dessen die Wahrscheinlichkeit für das Vorliegen einer krankheitsverursachenden Mutation besser abschätzen (•Tab. 3).

\section{Fazit für die Praxis}

Früh beginnende Demenzen umfassen alltagsrelevante kognitive Störungen mit Beginn zwischen dem 18. und 65. Lebensjahr. Obwohl Demenzen bei älteren Menschen deutlich häufiger sind, macht der Anteil jüngerer Patienten in spezialisierten Gedächtnisambulanzen bis zu einem Drittel der Fälle aus. Das differenzialdiagnostische Krankheitsspektrum ist deutlich breiter. Die häufigste Ursache einer früh beginnenden Demenz ist die AlzheimerKrankheit, die sich im Gegensatz zu älteren Patienten jedoch in bis zu zwei Drittel der Fälle als „atypische“ Varianten (PCA oder IvPPA) präsentiert. Die zweithäufigste Ursache ist die FTLD (als Verhaltensvariante oder PPA). Je jünger das Erkrankungsalter, desto höher die Wahrscheinlichkeit, dass es sich um eine sekundäre Demenz handelt. Um potenziell reversible Ursachen nicht zu übersehen und die Vielzahl klinischer Syndrome der primär neurodegenerativen Erkrankungen zu differenzieren, ist ein systematisches diagnostisches Vorgehen unabdingbar. Die Diagnostik sollte jedoch hypothesengeleitet sein und auf klinisch-syndromatologischen Einschätzungen beruhen, um unnötige und nicht indizierte Untersuchungen zu vermeiden. 


\section{Korrespondenzadresse}

\section{Dr. Dr. med. Matthias Pawlowski}

Klinik für Neurologie mit Institut für

Translationale Neurologie, Universitätsklinikum Münster

Albert-Schweitzer-Campus 1, Gebäude A1, 48149 Münster, Deutschland matthias.pawlowski@ukmuenster.de

\section{Prof. Dr. med. Thomas Duning}

Klinik für Neurologie mit Institut für

Translationale Neurologie, Universitätsklinikum Münster

Albert-Schweitzer-Campus 1, Gebäude A1, 48149 Münster, Deutschland thomas.duning@ukmuenster.de

Funding. Open Access funding provided by Projekt DEAL.

\section{Einhaltung ethischer Richtlinien}

Interessenkonflikt. M. Pawlowski, A. Johnen und T. Duning geben an, dass kein Interessenkonflikt besteht.

Für diesen Beitrag wurden von den Autoren keine Studien an Menschen oder Tieren durchgeführt. Für die aufgeführten Studien gelten die jeweils dort angegebenen ethischen Richtlinien.

Open Access. Dieser Artikel wird unter der Creative Commons Namensnennung 4.0 International Lizenz veröffentlicht, welche die Nutzung, Vervielfältigung, Bearbeitung, Verbreitung und Wiedergabe in jeglichem Medium und Format erlaubt, sofern Sie den/die ursprünglichen Autor(en) und die Quelle ordnungsgemäß nennen, einen Link zur Creative Commons Lizenz beifügen und angeben, ob Änderungen vorgenommen wurden.

Die in diesem Artikel enthaltenen Bilder und sonstiges Drittmaterial unterliegen ebenfalls der genannten Creative Commons Lizenz, sofern sich aus der Abbildungslegende nichts anderes ergibt. Sofern das betreffende Material nicht unter der genannten Creative Commons Lizenz steht und die betreffende Handlung nicht nach gesetzlichen Vorschriften erlaubt ist, ist für die oben aufgeführten Weiterverwendungen des Materials die Einwilligung des jeweiligen Rechteinhabers einzuholen.

Weitere Details zur Lizenz entnehmen Sie bitte der Lizenzinformation auf http://creativecommons.org/ licenses/by/4.0/deed.de.

\section{Literatur}

1. Alzheimer's Association (2017) 2017 Alzheimer's disease facts and figures. Alzheimers Dement 13:325-373

2. Bang J, Spina S, Miller BL (2015) Frontotemporal dementia. Lancet 386:1672-1682

3. Beck J, Rohrer JD, Campbell T et al (2008) A distinct clinical, neuropsychological and radiological phenotype is associated with progranulin gene mutations in a large UK series. Brain 131:706-720

4. Bickel H, Bürger K, Hampel H et al (2006) Presenile dementia in memory clinics - Incidence rates and clinical features. Nervenarzt 77:1079-1085

5. Brown J (2015) The use and misuse of short cognitive tests in the diagnosis of dementia. J Neurol Neurosurg Psychiatry 86:680-685

6. Clarfield AM (2003) The decreasing prevalence of reversible dementias: an updated meta-analysis. Arch Intern Med 163:2219-2229

7. Crutch SJ, Schott JM, Rabinovici GD et al (2017) Consensus classification of posterior cortical atrophy. Alzheimers Dement 13:870-884

8. Derby CA, Katz MJ, Lipton RB, Hall CB (2017) Trends in dementia incidence in a birth cohort analysis of the Einstein aging study. JAMA Neurol 74:1345-1351

9. Deuschl G, Maier W (2016) S3-Leitlinie Demenzen Leitlinien für Diagnostik und Therapie in der Neurologie, Bd. 128

10. Draper B, Withall A (2016) Young onset dementia Intern Med J 46:779-786

11. Folstein MF, Folstein SE, McHugh PR (1975) “Minimental state". A practical method for grading the cognitive state of patients for the clinician. JPsychiatr Res 12:189-198

12. Goldman JS, Van Deerlin VM (2018) Alzheimer's disease and frontotemporal dementia: the current state of genetics and genetic testing since the advent of next-generation sequencing. Mol Diagn Ther 22:505-513

13. Gorno-Tempini ML, Hillis $A E$, Weintraub $S$ et al (2011) Classification of primary progressive aphasia and its variants. Neurology 76:1006-1014

14. Harvey RJ, Skelton-Robinson M, Rossor MN (2003) The prevalence and causes of dementia in people under the age of 65 years. J Neurol Neurosurg Psychiatry 74:1206-1209

15. Huber SJ, Shuttleworth EC, Paulson GW et al (1986) Cortical vs subcortical dementia. Neuropsychological differences. Arch Neurol 43:392-394

16. Ikejima C, Yasuno F, Mizukami K et al (2009) Prevalence and causes of early-onset dementia in Japan: a population-based study. Stroke 40:2709-2714

17. Johnen A, Duning T (2017) Neuropsychologische Diagnostik in der Praxis. Was ist leitliniengerecht, was ist sinnvoll, was istmachbar? Klin Neurophysio 48:200-210

18. Kelley BJ, Boeve BF, Josephs KA (2008) Youngonset dementia: demographic and etiologic characteristics of 235 patients. Arch Neurol 65:1502-1508

19. Koopmans R, Rosness T (2014) Young onset dementia-What does the name imply? Int Psychogeriatr 26:1931-1933

20. Kvello-Alme M, Bråthen G, White LR, Sando SB (2019) The prevalence and subtypes of young onset dementia in central Norway: a populationbased study. J Alzheimers Dis 69:479-487

21. Lambert MA, Bickel $H$, Prince $M$ et al (2014) Estimating the burden of early onset dementia; systematic review of disease prevalence. Eur J Neurol 21:563-569

22. LansdallCJ, Coyle-Gilchrist ITS, Jones PSet al (2017) Apathy and impulsivity in frontotemporal lobar degeneration syndromes. Brain 140:1792-1807

23. Mendez MF (2019) Early-onset Alzheimer disease and its variants. Continuum (NY) 25:34-51

24. Mesulam M, Wicklund A, Johnson $\mathrm{N}$ et al (2008) Alzheimer and frontotemporal pathology in subsets of primary progressive aphasia. Ann Neurol 63:709-719
25. Nasreddine ZS, Phillips NA, Bédirian V et al (2005) The Montreal Cognitive Assessment, MoCA: a brief screening tool for mild cognitive impairment. J Am Geriatr Soc 53:695-699

26. Ossenkoppele R, Pijnenburg YAL, Perry DC et al (2015) The behavioural/dysexecutive variant of Alzheimer's disease: clinical, neuroimaging and pathological features. Brain 138:2732-2749

27. Pawlowski M, Meuth SG, Duning T (2017) Cerebrospinal fluid biomarkers in Alzheimer's disease-from brain starch to bench and bedside. Diagnostics 7:42

28. Rascovsky K, Hodges JR, Knopman D et al (2011) Sensitivity of revised diagnostic criteria for the behavioural variant of frontotemporal dementia. Brain 134:2456-2477

29. Reitz C, Brayne C, Mayeux R (2011) Epidemiology of Alzheimer disease. Nat Rev Neurol 7:137-152

30. Román GC, Erkinjuntti T, Wallin A et al (2002) Subcortical ischaemic vascular dementia. Lancet Neurol 1:426-436

31. Rossor MN, Fox NC, Mummery CJ et al (2010) The diagnosis of young-onset dementia. Lancet Neurol 9:793-806

32. Simrén J, Ashton NJ, Blennow K, Zetterberg H (2019) An update on fluid biomarkers for neurodegenerative diseases: recent success and challenges ahead. Curr Opin Neurobiol 61:29-39

33. Thomann AE, Goettel N, Monsch RJ et al (2018) The montreal cognitive assessment: normative data from a German-speaking cohort and comparison with international normative samples. JAlzheimers Dis 64:643-655

34. Warren JD, Fletcher PD, Golden HL (2012) The paradox of syndromic diversity in Alzheimer disease. Nat Rev Neurol 8:451-464

35. Withall A, Draper B, Seeher K, Brodaty H (2014) The prevalence and causes of younger onset dementia in Eastern Sydney, Australia. Int Psychogeriatr 26:1955-1965

36. Zucchella C, Federico A, Martini A et al (2018) Neuropsychological testing. Pract Neurol. https:// doi.org/10.1136/practneurol-2017-001743 